\title{
Existence of solutions for nonlinear Robin problems with the $p$-Laplacian and hemivariational inequality
}

Jing Zhang*

${ }^{\text {"Correspondence: }}$

zhjmath11@163.com

Y.Y. Tseng Functional Analysis

Research Center, Harbin Normal

University, Harbin, 150025, P.R. China

\begin{abstract}
In this paper, we show the existence of at least three nontrivial solutions for a nonlinear elliptic equation driven by the $p$-Laplacian with a nonsmooth potential (hemivariational inequality) and Robin boundary condition. Two of these solutions are of constant sign (one is positive, the other negative). We mainly use a variational approach together with a sub-sup solution method.
\end{abstract}

Keywords: $p$-Laplacian; nonsmooth potential; hemivariational inequality; sub-sup solution method; second deformation theorem

\section{Introduction}

Consider the problem

$$
\begin{cases}-\Delta_{p} x+\alpha|x|^{p-2} x \in \partial j(z, x), & z \in Z, \\ |\nabla x|^{p-2} \frac{\partial x}{\partial n}+b(z)|x|^{p-2} x=0, & z \in \partial Z,\end{cases}
$$

where $Z \subset \mathbb{R}^{N}$ is a bounded domain with $C^{2}$-boundary $\partial Z, \Delta_{p} x=\operatorname{div}\left(|\nabla x|^{p-2} \nabla x\right)(1<p<$ $\infty)$ is the $p$-Laplacian operator, $\alpha>0, b(z) \in L^{\infty}(\partial Z), b(z) \geq 0$, and $b(z) \neq 0$ on $\partial Z$. $j(z, x)$ is a measurable potential function on $Z \times \mathbb{R}$, which is locally Lipschitz in the $x \in \mathbb{R}, \partial j(z, x)$ stands for the generalized subdifferential of $x \mapsto j(z, x)$. Also $\frac{\partial x}{\partial n}$ denotes the outer normal derivative of $x$ with respect to $\partial Z$. The aim of this paper is to prove the existence of two constant sign solutions and furthermore prove the existence of at least three nontrivial solutions for problem (1.1).

A multiplicity of solutions for problems driven by the $p$-Laplacian has been obtained by Ambrosetti et al. [1] and Garcia Azorero et al. [2]. In these works, the authors deal with a right-hand side nonlinearity of the form $-\Delta_{p} x=\lambda|x|^{q-2} x+|x|^{r-2} x$ with $\lambda>0$ being a real parameter, $1<q<p<r<p^{*}\left(p^{*}=\frac{N p}{N-p}\right.$ if $p<N$; $p^{*}=+\infty$ otherwise) and prove the existence of positive and negative solutions. The question of the existence of a $p$-Laplacian Robin problem $-\Delta_{p} x+\alpha|x|^{p-2} x=j(z, x)$ was also present in the work of Zhang et al. [3] for $p=2$, the authors show that the Robin problem has at least four nontrivial solutions using a sub-sup solution method, the Fucík spectrum, the mountain pass theorem, and the degree theorem together. In the work of Zhang et al. $[4,5]$ for $p>2$, the authors show that the oscillating equations with the $p$-Laplacian Robin problem has infinitely many nontrivial

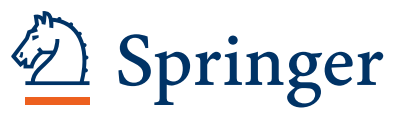

(c) 2014 Zhang; licensee Springer. This is an Open Access article distributed under the terms of the Creative Commons Attribution License (http://creativecommons.org/licenses/by/2.0), which permits unrestricted use, distribution, and reproduction in any medium, provided the original work is properly credited. 
solutions. In Anello [6] and Ricceri [7], the main tool is an abstract variational principle of Ricceri and its use is made possible by the hypothesis that $p>N$; by the fact that Sobolev space $W^{1, p}(Z)$ is compactly embedded in $C(\bar{Z})$, the authors obtain infinitely many weak solutions for $p$-Laplacian Neumann problem.

In all the aforementioned works, the nonlinearity is a Carathéodory function, a.e. $j(z, x)$ is continuously differentiable in the variable $x$. In Barletta and Papageorgiou [8], the authors consider a nonsmooth potential with an asymmetric behavior at $+\infty$ and at $-\infty$ to get two nontrivial solutions using degree methods. Also, in Dancer and Du [9], the authors use the critical point theory and a sub-sup solution method on smooth critical point theory.

In this paper, we use a combination of nonsmooth critical point theory with sub-sup solution methods. We also use the nonsmooth version of the second deformation theorem due to Corvellec [10]. Thus, we can extend the works of $[9,11-13]$ to a hemivariational inequality with the Robin boundary condition.

\section{Preliminaries}

Now we recall the subdifferential theory for locally Lipschitz functions and the corresponding nonsmooth critical point theory. Let $X$ be a Banach space and let $X^{*}$ be its topological dual. We denote by $\langle\cdot, \cdot\rangle$ the duality brackets for the pair $\left(X, X^{*}\right)$. The generalized directional derivative $\varphi^{0}(x ; h)$ of a locally Lipschitz function $\varphi: X \rightarrow \mathbb{R}$ at $x \in X$ along the direction $h \in X$ is defined as follows:

$$
\varphi^{0}(x ; h)=\limsup _{y \rightarrow x, \lambda \rightarrow 0} \frac{\varphi(y+\lambda h)-\varphi(y)}{\lambda} .
$$

It is well known that $\varphi^{0}(x ; \cdot)$ is sublinear continuous and it is the support function of a nonempty, convex, and $w^{*}$-compact set $\partial \varphi(x) \subseteq X^{*}$ defined by

$$
\partial \varphi(x)=\left\{x^{*} \in X^{*}:\left\langle x^{*}, h\right\rangle \leq \varphi^{0}(x ; h), \forall h \in X\right\} .
$$

The function $\partial \varphi(x)$ is the 'generalized subdifferential' of $\varphi$. If $\varphi \in C^{1}(X)$, then $\varphi$ is locally Lipschitz and $\partial \varphi(x)=\left\{\varphi^{\prime}(x)\right\}$. Moreover, if $\varphi$ is also convex, then $\partial \varphi(x)$ coincides with the subdifferential in the sense of convex analysis, $\partial_{c} \varphi(x)$, which is defined by

$$
\partial_{c} \varphi(x)=\left\{x^{*} \in X^{*}:\left\langle x^{*}, h\right\rangle \leq \varphi(x+h)-\varphi(x), \forall h \in X\right\}
$$

If $0 \in \partial \varphi(x)$, then we call $x \in X$ critical point of $\varphi$. It is easy to see that if $x \in X$ is a local minimum or a local maximum of $\varphi$, then $x \in X$ is a critical point of $\varphi$.

A locally Lipschitz function $\varphi$ satisfies the Palais-Smale condition at level $c \in \mathbb{R}$, if every sequence $\left\{x_{n}\right\}_{n \geq 1} \subseteq X$ satisfying $\varphi\left(x_{n}\right) \rightarrow c$ and $\inf \left\{\left\|x^{*}\right\|: x^{*} \in \partial \varphi\left(x_{n}\right)\right\} \rightarrow 0$ as $n \rightarrow \infty$ has a strongly convergent subsequence. If $\varphi$ satisfies the Palais-Smale condition at level $c \in \mathbb{R}$ for all $c \in \mathbb{R}$, then we say that it satisfies the Palais-Smale condition. For the details, we refer to [14].

In the following study, denote $R(z, x)=|\nabla x|^{p-2} \frac{\partial x}{\partial n}+b(z)|x|^{p-2} x$, and we will use the following spaces:

$$
W_{n}^{1, p}(Z)=\left\{x \in W^{1, p}(Z): \exists\left\{x_{n}\right\} \subset C^{\infty}(Z), x_{n} \rightarrow x \text { in } W^{1, p}(Z), R\left(z, x_{n}\right)=0, \forall z \in \partial Z\right\},
$$




$$
C_{n}^{1}(\bar{Z})=\left\{x \in C^{1}(\bar{Z}): R(z, x)=0, \forall z \in \partial Z\right\}
$$

Both are ordered Banach spaces, and we denote

$$
\begin{aligned}
& W^{+}=\left\{x \in W_{n}^{1, p}(Z): x(z) \geq 0 \text { a.e. } z \in Z\right\}, \\
& C^{+}=\left\{x \in C_{n}^{1}(\bar{Z}): x(z) \geq 0, \forall z \in \bar{Z}\right\}, \\
& \operatorname{int}\left(C^{+}\right)=\left\{x \in C^{+}: x(z)>0, \forall z \in Z\right\} .
\end{aligned}
$$

It is well known that the principal eigenfunction $e \in \operatorname{int}\left(C^{+}\right)$, so $\operatorname{int}\left(C^{+}\right) \neq \emptyset$.

Furthermore, define $u_{1}$ as the normalized principal eigenfunction of $\left(-\Delta_{p}, W_{n}^{1, p}(Z)\right)$ (see [15]). It is well known that $u_{1}(z) \geq 0$, a.e. $z \in Z$, from the nonlinear regularity $u_{1} \in C_{n}^{1}(\bar{Z})$ (see Di Benedetto [16], [17, Chapter IX]), furthermore $u_{1} \in \operatorname{int}\left(C^{+}\right)$by virtue of the strong maximum principle of Vazquez [18].

We give the following minimax characterization (see [19]), suited for our purpose.

Proposition 2.1 Let $S=W_{n}^{1, p}(Z) \cap \partial B_{1}$ and $\Gamma=\left\{\gamma \in C([0,1], S): \gamma(0)=-u_{1}, \gamma(1)=u_{1}\right\}$, where $\partial B_{1}=\left\{x \in L^{p}(Z):\|x\|_{p}=1\right\}$. Then the first eigenvalue $\lambda_{1}$ of $\left(-\Delta_{p}, W_{n}^{1, p}(Z)\right)$ equals

$$
\lambda_{1}=\inf _{\gamma \in \Gamma} \max _{t \in[0,1]}\|D \gamma(t)\|_{p}^{p}
$$

Next we recall the definitions of sub-sup solutions for problem (1.1).

(1) A function $\bar{x} \in W^{1, p}(Z)$ with $R(z, \bar{x}(z)) \geq 0$ is called a 'sup solution', if

$$
\begin{gathered}
\int_{Z}|D \bar{x}(z)|^{p-2}(D \bar{x}(z), D y(z)) d z+\alpha \int_{Z}|\bar{x}(z)|^{p-2}(\bar{x}(z), y(z)) d z \\
+\int_{\partial Z} b(z)|\bar{x}(z)|^{p-2}(\bar{x}(z), y(z)) d z \geq \int_{Z} u(z) y(z) d z
\end{gathered}
$$

for all $y \in W_{n}^{1, p}(Z), y(z) \geq 0$ a.e. on $Z$ and for some $u \in L^{q}(Z), u(z) \in \partial j(z, \bar{x}(z))$ a.e. on $Z$ for some $1<q<\frac{N p}{N-p}$ if $N>p, q=+\infty$ if $N \leq p$.

(2) A function $\underline{x} \in W^{1, p}(Z)$ with $R(z, \underline{x}(z)) \leq 0$ is called a 'sub-solution', if

$$
\begin{gathered}
\int_{Z}|D \underline{x}(z)|^{p-2}(D \underline{x}(z), D y(z)) d z+\alpha \int_{Z}|\underline{x}(z)|^{p-2}(\underline{x}(z), y(z)) d z \\
+\int_{\partial Z} b(z)|\underline{x}(z)|^{p-2}(\underline{x}(z), y(z)) d z \leq \int_{Z} u(z) y(z) d z
\end{gathered}
$$

for all $y \in W_{n}^{1, p}(Z), y(z) \geq 0$ a.e. on $Z$ and for some $u \in L^{q}(Z), u(z) \in j j(z, \underline{x}(z))$ a.e. on $Z$ for some $1<q<\frac{N p}{N-p}$ if $N>p, q=+\infty$ if $N \leq p$.

Finally we recall the following topological notion which is crucial in critical point theory.

Definition 2.2 [20] Let $S, Q$ be closed subsets of a Banach space $X, Q$ with relative boundary $\partial Q$. We say $S$ and $\partial Q$ link if

(1) $S \cap \partial Q=\emptyset$, and

(2) for any map $h \in C^{0}(X, X)$ such that $\left.h\right|_{\partial Q}=$ id we have $h(Q) \cap S \neq \emptyset$. 
From the definition, we give the following general minimax principle for the critical values of a locally Lipschitz function $\varphi$.

Proposition 2.3 [20] Suppose $\varphi$ is locally Lipschitz and satisfies the (PS)-condition. Consider closed subsets $S, Q \subset X$ and $Q$ with relative boundary $\partial Q$. Suppose

(1) $S$ and $\partial Q$ link,

(2) $\inf _{S} \varphi>\sup _{\partial Q} \varphi$.

Let

$$
\Gamma=\left\{h \in C^{0}(X, X):\left.h\right|_{\partial Q}=\mathrm{id}\right\} .
$$

Then the number

$$
\beta=\inf _{h \in \Gamma} \sup _{u \in Q} \varphi(h(u))
$$

defines a critical value $\beta \geq \inf _{S} \varphi$ of $\varphi$.

Remark 2.4 From the above general minimax principle, a nonsmooth version of the mountain pass theorem, the saddle point theorem, and the generalized mountain pass theorem are available by choosing the link sets appropriately (see [10, 14]).

The following result is the so-called 'second deformation theorem' for a nonsmooth setting. In fact, this result is due to Corvellec [10]. We give the following sets:

$$
\begin{aligned}
& K=\{x \in X: 0 \in \partial \varphi(x)\}, \\
& K_{c}=\{x \in X: 0 \in \partial \varphi(x), \varphi(x)=c\}, \\
& \varphi^{c}=\{x \in X: \varphi(x)<c\} .
\end{aligned}
$$

We know that $K, K_{c}$, and $\varphi^{c}$ are the critical set of $\varphi$, the critical set at level $c \in \mathbb{R}$ of $\varphi$, and the strict sublevel set of $\varphi$ at $c$, respectively.

Proposition 2.5 Let $X$ be a Banach space, $\varphi: X \rightarrow \mathbb{R}$ be locally Lipschitz satisfying the Palais-Smale condition. $a, b \in \mathbb{R}$ with $a<b$. Assume also that $K \cap \varphi^{-1}((a, b))=\emptyset$ and $K_{a}$ is a finite set containing only local minimizers of $\varphi$.

Then there exists a continuous deformation $\Phi:[0,1] \times \varphi^{b} \rightarrow \varphi^{b}$ such that

(1) $\Phi(t, x)=x$ for all $t \in[0,1], x \in K_{a}$,

(2) $\Phi\left(1, \varphi^{b}\right) \subseteq \varphi^{a} \cup K_{a}$,

(3) $\varphi(\Phi(t, x)) \leq \varphi(x)$ for all $t \in[0,1], x \in \varphi^{b}$.

Definition 2.6 [21] Let $X$ be a topological space and $A$ a subspace of $X$. A weak deformation retraction from $X$ to $A$ is a homology $F: X \times I \rightarrow X$ such that for all $x \in X$ and $a \in A$, we have $F(x, 0)=x, F(a, 1)=a$, and $F(x, 1) \in A$.

In particular, the set $\varphi^{a} \cup K_{a}$ is a weak deformation retract of $\varphi^{b}$.

We now recall another notion, which will be useful in the following. Suppose $W$ is a Banach space and $A: W \rightarrow W^{*}$ is a mapping, we say that $A$ is a type $(S)_{+}$if for every sequence $\left\{x_{n}\right\}_{n=1}^{\infty}$ such that $x_{n} \rightarrow x \in W$ and $\lim _{\sup _{n \rightarrow \infty}}\left\langle A\left(x_{n}\right), x_{n}-x\right\rangle \leq 0$. 
Considering the nonlinear mapping $A: W_{n}^{1, p}(Z) \rightarrow W_{n}^{1, p}(Z)^{*}$ defined for all $x \in W_{n}^{1, p}(Z)$ by

$$
\langle A(x), y\rangle=\int_{Z}|D x(z)|^{p-2} D x(z) \cdot D y(z) d z .
$$

We have the following result (see [8, Proposition 4.1]).

Proposition 2.7 The mapping (2.1) is continuous and of the type $(S)_{+}$.

Definition 2.8 [14] Given a functional $\varphi: W_{n}^{1, p}(Z) \rightarrow \mathbb{R}, x_{0} \in W_{n}^{1, p}(Z)$ is called a $W$-local minimizer of $\varphi$ if there exists $r>0$ satisfying for all $y \in W_{n}^{1, p}(Z)$ with $\|y\|_{W_{n}^{1, p}(Z)} \leq r$, we have

$$
\varphi\left(x_{0}\right) \leq \varphi\left(x_{0}+y\right)
$$

Definition $2.9[14] \quad x_{0} \in W_{n}^{1, p}(Z)$ is called a $C$-local minimizer of $\varphi$ if there exists $r>0$ satisfying for all $y \in C_{n}^{1}(\bar{Z})$ with $\|y\|_{C_{n}^{1}(\bar{Z})} \leq r$, we have

$$
\varphi\left(x_{0}\right) \leq \varphi\left(x_{0}+y\right)
$$

As the study of problems like (1.1) is reduced to seeking the critical points of corresponding energy functional on $W_{n}^{1, p}(Z)$ or on $C_{n}^{1}(\bar{Z})$, in this section we introduce the notations used along the paper together with the main abstract results that we will use later on for a $C$-local minimizer to be a $W$-local minimizer. Such a result for $p>2$ was first proved in [2]. Then it has been extended to the Neumann boundary condition and a nonsmooth potential by [8].

We denote $\psi: W_{n}^{1, p}(Z) \rightarrow \mathbb{R}$ for all $x \in W_{n}^{1, p}(Z)$

$$
\psi(x)=\frac{1}{p}\|D x\|_{p}^{p}+\frac{1}{p} \int_{\partial Z} b(z)|x|^{p} d s-\int_{Z} j(z, x(z)) d z .
$$

From Clarke [22, pp.32-34], we know that $\psi$ is locally Lipschitz. By [12], we know that if we let $x_{0} \in W_{n}^{1, p}(Z)$ be a $C$-local minimizer of $\psi$, then $x_{0} \in C_{n}^{1}(\bar{Z})$ and it is a $W$-local minimizer of $\psi$.

\section{Solutions of constant sign}

In this section, by using a sub-sup solution method, we get two solutions of (1.1) with constant sign, one positive and the other negative.

Our general assumptions on the nonsmooth potential $j(z, x)$ are the following:

$A(j) \quad$ (i) $z \mapsto j(z, x)$ is measurable for all $x \in \mathbb{R}$;

(ii) $x \mapsto j(z, x)$ is locally Lipschitz for a.e. $z \in Z$;

(iii) $|u| \leq \gamma(z)+C|x|^{p-1}$ for a.e. $z \in Z$, all $x \in \mathbb{R}, u \in \partial j(z, x)$, with $\gamma \in L^{\infty}(Z)_{+}$and $C>0$;

(iv) $\lim \sup _{|x| \rightarrow \infty} \frac{u}{|x|^{p-2 x}} \leq \omega(z)$ for a.e. $z \in Z$, all $u \in \partial j(z, x)$, with $\omega \in L^{\infty}(Z)_{+}$ satisfying $\omega(z) \leq \alpha$ a.e. in $Z$ and $\omega(z)<\alpha$ in some set of positive measure; 
(v) $\eta(z)+\alpha \leq \liminf _{x \rightarrow 0} \frac{u}{|x|^{p-2} x}$ for a.e. $z \in Z$, all $u \in \partial j(z, x)$, with $\eta \in L^{\infty}(Z)_{+}$ satisfying $\lambda_{1} \leq \eta(z)$ a.e. in $Z$ and $\lambda_{1}<\eta(z)$ in some set of positive measure, $\lambda_{1}$ is the first eigenvalue of $-\Delta_{p}$ with Robin boundary condition;

(vi) $u x \geq 0$ for a.e. $z \in Z$, all $x \in \mathbb{R}, u \in \partial j(z, x)$.

Theorem 3.1 Assume that $A(j)(\mathrm{i})$-(vi) hold. Problem (1.1) has at least two solutions $x_{0} \in$ $\operatorname{int}\left(C_{+}\right)$and $x_{*} \in-\operatorname{int}\left(C_{+}\right)$.

Example The following potential function $j$ satisfies assumptions $A(j)$ (for the sake of simplicity we drop the $z$-dependence):

$$
j(x)= \begin{cases}\frac{\eta+\alpha}{p}|x|^{p}, & |x| \leq 1, \\ \frac{\omega}{p}|x|^{p}+C \ln |x|^{p}+\frac{\eta+\alpha-\omega}{p}, & |x|>1,\end{cases}
$$

where $0<\omega<\alpha, \eta>\lambda_{1}$, and $C>0$. Note that, if $C=\frac{\eta+\alpha-\omega}{p}$, then $j \in C^{1}(\mathbb{R})$.

Note that

$$
\partial j(x)= \begin{cases}{[-\omega-p C,-\eta-\alpha],} & x=-1, \\ (\eta+\alpha)|x|^{p-2} x, & |x| \leq 1, \\ 0, & x=0, \\ \omega|x|^{p-2} x+p C \frac{x}{|x|^{2}}, & |x|>1, \\ {[\eta+\alpha, \omega+p C],} & x=1 .\end{cases}
$$

It is easy to see that $j$ satisfies $A(j)$ (i)-(iii), (vi). For all $u \in \partial j(x)$, we have

$$
\limsup _{|x| \rightarrow \infty} \frac{u}{|x|^{p-2} x} \leq \omega
$$

and

$$
\eta+\alpha \leq \liminf _{x \rightarrow 0} \frac{u}{|x|^{p-2} x} .
$$

Then the potential function $j$ satisfies assumptions $A(j)$.

Remark 3.2 In fact, problem (1.1) has the trivial solution $0 \in \partial j(z, 0)$ for a.e. $z \in Z$ according to assumption $A(j)(\mathrm{vi})$ and the upper semicontinuity of the subdifferential $\partial j(z, \cdot)$ (see Clarke [22]). What we are interesting in is whether it has nontrivial solutions.

We introduce a useful extension of the notion of maximal monotonicity (see [14, p.83]).

Definition 3.3 Let $X$ be a reflexive Banach space and $A: X \rightarrow 2^{X^{*}}$ an operator. We say that $A$ is pseudomonotone if

(1) $A$ has nonempty, bounded and convex values;

(2) $A$ is upper semicontinuous for every finite dimensional subspace of $X$ into $X^{*}$;

(3) if $x_{n} \rightarrow x$ in $X, x_{n}^{*} \in A\left(x_{n}\right)$, and $\lim \sup _{n \rightarrow+\infty}\left\langle x_{n}^{*}, x_{n}-x\right\rangle_{X} \leq 0$, then for every $y \in X$, there exists $u^{*}(y) \in A(x)$, such that

$$
\left\langle u^{*}(y), x-y\right\rangle_{X} \leq \liminf _{n \rightarrow+\infty}\left\langle x_{n}^{*}, x_{n}-y\right\rangle_{X}
$$


Definition 3.4 [14] A is said to be demicontinuous on $X$ if $\left\{x_{n}\right\} \subset X$ and $x_{n} \rightarrow x \in X$ together imply $A\left(x_{n}\right) \rightarrow A(x)$.

It is well known that (1.1) is the Euler-Lagrange equation of the functional $\varphi: W_{n}^{1, p}(Z) \rightarrow$ $\mathbb{R}$,

$$
\varphi(x)=\frac{1}{p}\|D x\|_{p}^{p}+\frac{\alpha}{p}\|x\|_{p}^{p}+\frac{1}{p} \int_{\partial Z} b(z)|x|^{p} d s-\int_{Z} j(z, x(z)) d z, \quad \forall x \in W_{n}^{1, p}(Z) .
$$

We introduce the truncation function $v_{+}: \mathbb{R} \rightarrow \mathbb{R}_{+}$by

$$
v_{+}(x)= \begin{cases}x, & x>0 \\ 0, & x \leq 0\end{cases}
$$

then define the locally Lipschitz functional $\varphi_{+}: W_{n}^{1, p}(Z) \rightarrow \mathbb{R}$ by

$$
\varphi_{+}(x)=\frac{1}{p}\|D x\|_{p}^{p}+\frac{\alpha}{p}\|x\|_{p}^{p}+\frac{1}{p} \int_{\partial Z} b(z)|x|^{p} d s-\int_{Z} j_{+}(z, x(z)) d z, \quad \forall x \in W_{n}^{1, p}(Z),
$$

where $j_{+}(z, x)=j\left(z, v_{+}(x)\right)$ for all $z \in \mathbb{R}, x \in \mathbb{R}$, which is locally Lipschitz.

We consider the nonlinear Robin problem for given $\varepsilon>0$ and $\delta_{\varepsilon}(z) \in L^{\infty}(Z)_{+}, \delta_{\varepsilon} \neq 0$ :

$$
\begin{cases}-\Delta_{p} x+\alpha|x|^{p-2} x=(\omega(z)+\varepsilon)|x|^{p-2} x+\delta_{\varepsilon}(z), & z \in Z, \\ |\nabla x|^{p-2} \frac{\partial x}{\partial n}+b(z)|x|^{p-2} x=0, & z \in \partial Z .\end{cases}
$$

Define the mapping $I: W_{n}^{1, p}(Z) \rightarrow W_{n}^{1, p}(Z)^{*}$ for all $x, y \in W_{n}^{1, p}(Z)$ by

$$
\begin{aligned}
\langle I(x), y\rangle= & \int_{Z}|D x(z)|^{p-2} D x(z) \cdot D y(z) d z+\alpha \int_{Z}|x(z)|^{p-2} x(z) \cdot y(z) d z \\
& +\int_{\partial Z} b(z)|x(z)|^{p-2} x(z) \cdot y(z) d s .
\end{aligned}
$$

It is well known that $I$ is strictly monotone and demicontinuous, furthermore, maximal monotone (see [23]). We denote $K_{\varepsilon}: L^{p}(Z) \rightarrow L^{p^{\prime}}(Z)\left(\frac{1}{p}+\frac{1}{p^{\prime}}=1\right)$ and we have

$$
K_{\varepsilon}(x)(\cdot)=(\omega(\cdot)+\varepsilon)|x(\cdot)|^{p-2} x(\cdot)
$$

which is bounded and continuous. Then the mapping $I(x)-K_{\varepsilon}(x)$ is pseudomonotone from $W_{n}^{1, p}(Z)$ into $W_{n}^{-1, p^{\prime}}(Z)$, in fact, $W_{n}^{1, p}(Z) \hookrightarrow L^{p}(Z)$ is compact embedding and $K_{\varepsilon}$ : $W_{n}^{1, p}(Z) \rightarrow L^{p^{\prime}}(Z)$ is completely continuous.

Next, we will show that (3.1) has a solution $\bar{x} \in \operatorname{int}\left(C_{+}\right)$.

Lemma 3.5 Let $\omega \in L^{\infty}(Z)_{+}$satisfy $\omega(z) \leq \alpha$ a.e. in $Z$ and $\omega(z)<\alpha$ in some set of positive measure. Then (3.1) has a solution $\bar{x} \in \operatorname{int}\left(C_{+}\right)$for $\varepsilon>0$ small enough.

Proof First, we claim that there exists $\xi_{0}>0$ such that

$$
J(x)=\|D x\|_{p}^{p}+\alpha\|x\|_{p}^{p}+\int_{\partial Z} b(z)|x|^{p} d s-\int_{Z} \omega(z)|x(z)|^{p} d z \geq \xi_{0}\|x\|_{p}^{p}, \quad \forall x \in W_{n}^{1, p}(Z) .
$$


In fact, from assumption $b \geq 0$, we know that $J(x) \geq 0$, for all $x \in W_{n}^{1, p}(Z)$. Suppose the conclusion is false, we have $x_{n} \in W_{n}^{1, p}(Z), J\left(x_{n}\right)<\frac{1}{n}\left\|x_{n}\right\|_{p}^{p}, x_{n} \neq 0$. If we set $x_{n}^{\prime}=\frac{x_{n}}{\left\|x_{n}\right\|}$, then $J\left(x_{n}^{\prime}\right)<\frac{1}{n}\left(J\right.$ is $p$-homogeneous). We may assume $x_{n}^{\prime} \rightarrow x$ in $W_{n}^{1, p}(Z), x_{n}^{\prime} \rightarrow x$ in $L^{p}(Z)$ by passing to a subsequence if necessary. Then

$$
\begin{aligned}
& \|x\|_{p}^{p}=\lim _{n \rightarrow \infty}\left\|x_{n}^{\prime}\right\|_{p}^{p}, \quad\|D x\|_{p}^{p} \leq \liminf _{n \rightarrow \infty}\left\|D x_{n}^{\prime}\right\|_{p}^{p}, \\
& \int_{\partial Z} b(z)|x|^{p} d s-\int_{Z} \omega(z)|x(z)|^{p} d z=\lim _{n \rightarrow \infty}\left(\int_{\partial Z} b(z)\left|x_{n}^{\prime}\right|^{p} d s-\int_{Z} \omega(z)\left|x_{n}^{\prime}(z)\right|^{p} d z\right) .
\end{aligned}
$$

So, by passing to the limit of $J$, we have

$$
\|D x\|_{p}^{p}+\alpha\|x\|_{p}^{p}+\int_{\partial Z} b(z)|x|^{p} d s-\int_{Z} \omega(z)|x(z)|^{p} d z \leq 0 .
$$

This implies

$$
\|D x\|_{p}^{p} \leq \int_{Z}(\omega(z)-\alpha)|x(z)|^{p} d z-\int_{\partial Z} b(z)|x|^{p} d s \leq 0 .
$$

Hence, we have $x(z)=C$ for a.e. $z \in Z$ where $C \in \mathbb{R}$. In fact, $C=0$, if not, from the above inequality,

$$
0 \leq|C|^{p}\left[\int_{Z}(\omega(z)-\alpha) d z-\int_{\partial Z} b(z) d s\right]<0 .
$$

It produces a contradiction. On the other hand,

$$
\left\|D x_{n}^{\prime}\right\|_{p}^{p}=J\left(x_{n}^{\prime}\right)-\alpha\left\|x_{n}^{\prime}\right\|_{p}^{p}-\int_{\partial Z} b(z)\left|x_{n}^{\prime}\right|^{p} d s-\int_{Z} \omega(z)\left|x_{n}^{\prime}(z)\right|^{p} d z .
$$

We have $\left\|D x_{n}^{\prime}\right\|_{p} \rightarrow 0$, together with $x_{n} \rightarrow x$ in $L^{p}(Z)$, so $x_{n} \rightarrow 0$ in $W_{n}^{1, p}(Z)$, but $\left\|x_{n}\right\|_{W_{n}^{1, p}(Z)}=1, n \in Z$. So the assumption is false, we have the conclusion.

For all $x \in W_{n}^{1, p}(Z)$, from the above discussion, we get

$$
\begin{aligned}
\left\langle I(x)-K_{\varepsilon}(x), x\right\rangle & =\|D x\|_{p}^{p}+\alpha\|x\|_{p}^{p}+\int_{\partial Z} b(z)|x|^{p} d s-\int_{Z} \omega(z)|x(z)|^{p} d z-\varepsilon\|x\|_{p}^{p} \\
& \geq\left(\varepsilon_{0}-\varepsilon\right)\|x\|_{p}^{p} .
\end{aligned}
$$

So if $\varepsilon<\varepsilon_{0}$ small enough, we have $I(\cdot)-K_{\varepsilon}(\cdot)$ is coercive. But a pseudomonotone coercive operator is surjective (see [23, Theorem 9.57]), for $\delta_{\varepsilon}$, we can find $\bar{x} \in W_{n}^{1, p}(Z)$ such that

$$
I(\bar{x})-K_{\varepsilon}(\bar{x})=\delta_{\varepsilon} .
$$

That is,

$$
\left\{\begin{array}{l}
-\Delta_{p} \bar{x}+\alpha|\bar{x}|^{p-2} \bar{x}=(\omega(z)+\varepsilon)|\bar{x}|^{p-2} \bar{x}+\delta_{\varepsilon}(z), \quad z \in Z, \\
|\nabla \bar{x}|^{p-2} \frac{\partial \bar{x}}{\partial n}+b(z)|\bar{x}|^{p-2} \bar{x}=0, \quad z \in \partial Z .
\end{array}\right.
$$

It follows that $\bar{x} \in W_{n}^{1, p}(Z)$ is a solution of (3.1). 
Next we show $\bar{x} \in \operatorname{int}\left(C_{+}\right)$. Take $-\bar{x}_{-}=-\max \{-\bar{x}, 0\} \in W_{n}^{1, p}(Z)$ for $\delta_{\varepsilon} \geq 0$, then

$$
\begin{aligned}
\left\langle I(\bar{x})-K_{\varepsilon}(\bar{x}),-\bar{x}_{-}\right\rangle= & -\left\|D \bar{x}_{-}\right\|_{p}^{p}-\alpha\left\|\bar{x}_{-}\right\|_{p}^{p}-\int_{\partial Z} b(z)\left|\bar{x}_{-}\right|^{p} d s \\
& +\int_{Z} \omega(z)\left|\bar{x}_{-}(z)\right|^{p} d z+\varepsilon\left\|\bar{x}_{-}\right\|_{p}^{p} \geq 0 .
\end{aligned}
$$

So

$$
\varepsilon_{0}\left\|\bar{x}_{-}\right\|_{p}^{p} \leq\left\|D \bar{x}_{-}\right\|_{p}^{p}+\alpha\left\|\bar{x}_{-}\right\|_{p}^{p}+\int_{\partial Z} b(z)\left|\bar{x}_{-}\right|^{p} d s-\int_{Z} \omega(z)\left|\bar{x}_{-}(z)\right|^{p} d z \leq \varepsilon\left\|\bar{x}_{-}\right\|_{p}^{p} .
$$

But $\varepsilon<\varepsilon_{0}$, we have $\bar{x}_{-}=0$, that is, $\bar{x} \geq 0$. Since $\delta_{\varepsilon}>0$, from (3.2), we have $\bar{x} \neq 0$ and $\bar{x} \in C_{n}^{1}(\bar{Z})$ (nonlinear regularity theorem, see [24]), furthermore, $\Delta_{p} \bar{x} \leq 0$ on $Z$, so $\bar{x} \in$ $\operatorname{int}\left(C_{+}\right)$.

Now we prove that the solution $\bar{x}$ of (3.1) is a strict sup solution of (1.1) for $\varepsilon>0$ small enough.

Lemma 3.6 Let assumptions $A(j)(\mathrm{i})$-(iv) hold. Then the solution $\bar{x}$ of (3.1) is a strict sup solution of (1.1) for $\varepsilon>0$ small enough.

Proof From $A(j)$ (iv), for given $\varepsilon>0$, we can find $M_{1}>0$, such that for all $z \in Z, x \geq M_{1}$, $u \in \partial j(z, x)$, we have

$$
\frac{u}{x^{p-1}} \leq \omega(z)+\varepsilon
$$

From $A(j)\left(\right.$ iii), we can find $\delta_{\varepsilon} \in L^{\infty}(Z)_{+}, \delta_{\varepsilon} \neq 0$, such that for all $z \in Z, x \in\left[0, M_{1}\right], u \in$ $\partial j(z, x)$, we have

$$
u<\delta_{\varepsilon}(z)
$$

So for all $z \in Z, x \geq 0, u \in \partial j(z, x)$, we have

$$
u<(\omega(z)+\varepsilon) x^{p-1}+\delta_{\varepsilon}(z)
$$

From Lemma 3.5, we see that (3.1) has a solution $\bar{x} \in \operatorname{int}\left(C_{+}\right)$, so when $\varepsilon<\varepsilon_{0}$ small enough, for all $z \in Z, x \in L^{p^{\prime}}(Z)_{+}, u \in \partial j(z, \bar{x}(z))$, we have

$$
u<(\omega(z)+\varepsilon) \bar{x}^{p-1}+\delta_{\varepsilon}(z)
$$

that is,

$$
u<-\Delta_{p} \bar{x}+\alpha|\bar{x}|^{p-2} \bar{x}
$$

and from the definition of a sup solution, we know that $\bar{x}$ is a sup solution of (1.1). 
Remark 3.7 We have found a sup solution of $(1.1)$ and $\partial j(z, 0)=\{0\}$ a.e. on $Z$, we also find $\underline{x} \equiv 0$ is a sub-solution of (1.1). Define the set

$$
W=\left\{x \in W_{n}^{1, p}(Z): 0 \leq x(z) \leq \bar{x}(z) \text {, a.e. on } Z\right\} .
$$

Next, we will find a nontrivial solution of (1.1) in $W$.

\section{Proof of Theorem 3.1}

Step 1: Claim: We can find $x_{0} \in W$ which is a local minimizer of $\varphi_{+}$and of $\varphi$.

From the discussion of Lemma 3.6, for a.e. $z \in Z$, all $x \geq 0, u \in \partial j_{+}(z, x)=\partial j(z, x)$, we have

$$
u<(\omega(z)+\varepsilon) x^{p-1}+\delta_{\varepsilon}(z)
$$

Furthermore, for a.e. $z \in Z$, all $x \geq 0$, from assumptions $A(j)$ (i), (ii),

$$
\frac{d}{d x} j_{+}(z, x)<(\omega(z)+\varepsilon) x^{p-1}+\delta_{\varepsilon}(z)
$$

then for a.e. $z \in Z$, all $x \geq 0$, we have

$$
j_{+}(z, x)<\frac{1}{p}(\omega(z)+\varepsilon)|x|^{p}+\delta_{\varepsilon}(z)|x| .
$$

So, for some $C>0$, we have

$$
\begin{aligned}
\varphi_{+}(x) & =\frac{1}{p}\|D x\|_{p}^{p}+\frac{\alpha}{p}\|x\|_{p}^{p}+\frac{1}{p} \int_{\partial Z} b(z)|x|^{p} d s-\int_{Z} j_{+}(z, x(z)) d z \\
& >\frac{1}{p}\|D x\|_{p}^{p}+\frac{\alpha}{p}\|x\|_{p}^{p}+\frac{1}{p} \int_{\partial Z} b(z)|x|^{p} d s-\frac{1}{p} \int_{Z} \omega(z)|x(z)|^{p} d z-\frac{\varepsilon}{p}\|x\|_{p}^{p}-C\|x\|_{p} \\
& \geq \frac{1}{p}\left(\varepsilon_{0}-\varepsilon\right)\|x\|_{p}^{p}-C\|x\|_{p} .
\end{aligned}
$$

Because of $\varepsilon<\varepsilon_{0}, p>1$, we see that $\varphi_{+}$is coercive, and together with $\varphi_{+}$weakly lower semicontinuous on $W$. Thus by the Weierstrass theorem, we can find $x_{0} \in W$, satisfying

$$
\varphi_{+}\left(x_{0}\right)=\inf _{W} \varphi_{+} \cdot
$$

We claim that $x_{0} \neq 0$. In fact, from assumption $A(j)(\mathrm{v})$, we see that, for given $\varepsilon>0$, we can find some $\delta>0$, for a.e. $z \in Z$, all $x \in[0, \delta], u \in \partial j_{+}(z, x)=\partial j(z, x)$,

$$
\frac{u}{x^{p-1}} \geq \eta(z)+\alpha-\varepsilon
$$

then for a.e. $z \in Z$ and all $x \in[0, \delta]$, we get

$$
j_{+}(z, x) \geq \frac{1}{p}(\eta(z)+\alpha-\varepsilon) x^{p} .
$$


Furthermore, let $e_{1}$ be the first eigenfunction of Robin problem of $-\Delta_{p}$ (see [15]), then for $\bar{x} \in \operatorname{int}\left(C^{+}\right)$, we can find $\theta>0$, such that

$$
\theta e_{1}(z) \leq \min \{\bar{x}(z), \delta\}, \quad \forall z \in \bar{Z}
$$

Then $\theta e_{1} \in \operatorname{int}\left(C^{+}\right)$, and

$$
\begin{aligned}
\varphi_{+}\left(\theta e_{1}\right) & =\frac{\theta^{p}}{p}\left\|D e_{1}\right\|_{p}^{p}+\frac{\alpha \theta^{p}}{p}\left\|e_{1}\right\|_{p}^{p}+\frac{\theta^{p}}{p} \int_{\partial Z} b(z)\left|e_{1}\right|^{p} d s-\int_{Z} j_{+}\left(z, \theta e_{1}(z)\right) d z \\
& \leq \frac{\theta^{p}}{p}\left\|D e_{1}\right\|_{p}^{p}-\frac{\theta^{p}}{p} \int_{Z} \eta(z)\left|e_{1}\right|^{p} d z+\frac{\theta^{p}}{p} \int_{\partial Z} b(z)\left|e_{1}\right|^{p} d s+\frac{\varepsilon \theta^{p}}{p}\left\|e_{1}\right\|_{p}^{p} \\
& =\frac{\theta^{p}}{p}\left[\int_{Z}\left(\lambda_{1}-\eta(z)\right)\left|e_{1}\right|^{p} d z+\varepsilon\left\|e_{1}\right\|_{p}^{p}\right] .
\end{aligned}
$$

From assumption $A(j)(\mathrm{v})$ and $e_{1}>0$, we have

$$
\int_{Z}\left(\lambda_{1}-\eta(z)\right)\left|e_{1}\right|^{p} d z<0
$$

If we choose $\varepsilon$ small enough, we can get $\varphi_{+}\left(\theta e_{1}\right)<0$ for all $\theta>0$ small enough. So, we have

$$
\varphi_{+}\left(x_{0}\right)=\inf _{W} \varphi_{+} \leq \varphi_{+}\left(\theta e_{1}\right)<0=\varphi_{+}(0)
$$

then we have $x_{0} \neq 0, x_{0} \in W$.

Step 2: The local minimizer of $\varphi_{+}, x_{0} \in W_{n}^{1, p}(Z)$ is a nontrivial solution of (1.1).

Firstly, we claim that $x_{0}$ is also a local $W_{n}^{1, p}(Z)$-minimizer of $\varphi_{+}$. In fact, the nonlinear regularity theory (see for example [24]) assures that $x_{0} \in C^{1}(\bar{Z})$. Hence, as the boundary relation is understood in a pointwise sense and we get $x_{0} \in C_{n}^{1}(\bar{Z})$, also, by $x_{0} \neq 0, x_{0} \geq 0$, and the nonlinear strong maximum principle of Vazquez, $x_{0} \in \operatorname{int}\left(C_{+}\right), \bar{x}-x_{0} \in \operatorname{int}\left(C_{+}\right)$. So we can find $\delta>0$ satisfying

$$
\begin{aligned}
& B_{\delta}\left(x_{0}\right)=\left\{x \in C_{n}^{1}(\bar{Z}):\left\|x-x_{0}\right\|_{C_{n}^{1}(\bar{Z})}<\delta\right\} \subseteq \operatorname{int}\left(C_{+}\right), \\
& B_{\delta}\left(\bar{x}-x_{0}\right)=\left\{x \in C_{n}^{1}(\bar{Z}):\left\|x-\left(\bar{x}-x_{0}\right)\right\|_{C_{n}^{1}(\bar{Z})}<\delta\right\} \subseteq \operatorname{int}\left(C_{+}\right) .
\end{aligned}
$$

Then

$$
x_{0}+B_{\delta} \subseteq \operatorname{int}\left(C_{+}\right), \quad \bar{x}-x_{0}+B_{\delta} \subseteq \operatorname{int}\left(C_{+}\right) .
$$

So, $x_{0}$ is also a local minimizer of $\varphi_{+}$on $C_{n}^{1}(\bar{Z})$; also from [24], $x_{0}$ is also a local $W_{n}^{1, p}(Z)$ minimizer of $\varphi_{+}$and of $\varphi$ too.

Also, from [25], there exists $\omega(z) \in \partial j_{+}\left(z, x_{0}(z)\right)=\partial j\left(z, x_{0}(z)\right), u \in L^{p^{\prime}}(Z)$ satisfying

$$
0 \leq\left\langle I\left(x_{0}\right), y-x_{0}\right\rangle-\int_{Z} u(z)\left(y-x_{0}\right)(z) d z, \quad \forall y \in W .
$$


Using

$$
y(z)= \begin{cases}\bar{x}(z), & z \in\left\{\bar{x} \leq x_{0}+\varepsilon v\right\}=A, \\ x_{0}(z)+\varepsilon v(z), & z \in\left\{0<x_{0}+\varepsilon v<\bar{x}\right\}=B, \\ 0, & z \in\left\{x_{0}+\varepsilon v \leq 0\right\}=C .\end{cases}
$$

We have $y \in W$ for all $v \in W_{n}^{1, p}(Z), \varepsilon>0$, then we have

$$
\begin{aligned}
& 0 \leq \int_{A}\left|D x_{0}\right|^{p-2}\left\langle D x_{0}, D\left(\bar{x}-x_{0}\right)\right\rangle d z+\alpha \int_{A}\left|x_{0}\right|^{p-2}\left\langle x_{0}, \bar{x}-x_{0}\right\rangle d z-\int_{A} u\left(\bar{x}-x_{0}\right) d z \\
& +\varepsilon \int_{B}\left|D x_{0}\right|^{p-2}\left\langle D x_{0}, D v\right\rangle d z+\alpha \int_{B}\left|x_{0}\right|^{p-2}\left\langle x_{0}, \varepsilon v\right\rangle d z-\int_{B} u(\varepsilon v) d z \\
& +\int_{\partial B} b(z)\left|x_{0}\right|^{p-2}\left\langle x_{0}, \varepsilon v\right\rangle d s \\
& -\int_{C}\left|D x_{0}\right|^{p} d z-\alpha \int_{C}\left|x_{0}\right|^{p} d z+\int_{C} u x_{0} d z-\int_{\partial C} b(z)\left|x_{0}\right|^{p} d s \\
& +\int_{\partial A} b(z)\left|x_{0}\right|^{p-2}\left\langle x_{0}, \bar{x}-x_{0}\right\rangle d s \\
& =\varepsilon \int_{Z}\left|D x_{0}\right|^{p-2}\left\langle D x_{0}, D v\right\rangle d z+\varepsilon \alpha \int_{Z}\left|x_{0}\right|^{p-2}\left\langle x_{0}, v\right\rangle d z-\varepsilon \int_{Z} u v d z \\
& +\varepsilon \int_{\partial Z} b(z)\left|x_{0}\right|^{p-2}\left\langle x_{0}, v\right\rangle d s \\
& -\int_{C}\left|D x_{0}\right|^{p} d z-\alpha \int_{C}\left|x_{0}\right|^{p} d z-\varepsilon \int_{C}\left|D x_{0}\right|^{p-2}\left\langle D x_{0}, D v\right\rangle d z-\int_{\partial C} b(z)\left|x_{0}\right|^{p} d s \\
& -\int_{A}|D \bar{x}|^{p-2}\left\langle D \bar{x}, D\left(x_{0}+\varepsilon v-\bar{x}\right)\right\rangle d z-\alpha \int_{A}|\bar{x}|^{p-2}\left\langle\bar{x}, x_{0}+\varepsilon v-\bar{x}\right\rangle d z \\
& +\int_{A} \bar{u}\left(x_{0}+\varepsilon v-\bar{x}\right) d z \\
& -\int_{\partial A} b(z)|\bar{x}|^{p-2}\left\langle\bar{x}, x_{0}+\varepsilon v-\bar{x}\right\rangle d s+\int_{C} u\left(x_{0}+\varepsilon v\right) d z+\int_{A}(\bar{u}-u)\left(\bar{x}-x_{0}-\varepsilon v\right) d z \\
& +\int_{A}\left\langle|D \bar{x}|^{p-2} D \bar{x}-\left|D x_{0}\right|^{p-2} D x_{0}, D\left(x_{0}-\bar{x}\right)\right\rangle d z+\alpha \int_{A}\left\langle|\bar{x}|^{p-2} \bar{x}-\left|x_{0}\right|^{p-2} x_{0}, x_{0}-\bar{x}\right\rangle d z \\
& +\int_{\partial A} b(z)\left\langle|\bar{x}|^{p-2} \bar{x}-\left|x_{0}\right|^{p-2} x_{0}, x_{0}-\bar{x}\right\rangle d s+\varepsilon \int_{C}\left\langle|D \bar{x}|^{p-2} D \bar{x}-\left|D x_{0}\right|^{p-2} D x_{0}, D v\right\rangle d z \\
& -\varepsilon \int_{\partial A} b(z)\left|x_{0}\right|^{p-2}\left\langle x_{0}, v\right\rangle d s-\varepsilon \int_{\partial C} b(z)\left|x_{0}\right|^{p-2}\left\langle x_{0}, v\right\rangle d s+\varepsilon \int_{\partial A} b(z)|\bar{x}|^{p-2}\langle\bar{x}, v\rangle d s \\
& -\varepsilon \alpha \int_{A}\left|x_{0}\right|^{p-2}\left\langle x_{0}, v\right\rangle d x-\varepsilon \alpha \int_{C}\left|x_{0}\right|^{p-2}\left\langle x_{0}, v\right\rangle d x+\varepsilon \alpha \int_{A}|\bar{x}|^{p-2}\langle\bar{x}, v\rangle d x .
\end{aligned}
$$

From the monotonicity of $I$, we have

$$
\begin{aligned}
& \int_{A}\left\langle|D \bar{x}|^{p-2} D \bar{x}-\left|D x_{0}\right|^{p-2} D x_{0}, D\left(x_{0}-\bar{x}\right)\right\rangle d z+\alpha \int_{A}\left\langle|\bar{x}|^{p-2} \bar{x}-\left|x_{0}\right|^{p-2} x_{0}, x_{0}-\bar{x}\right\rangle d z \\
& \quad+\int_{\partial A} b(z)\left\langle|\bar{x}|^{p-2} \bar{x}-\left|x_{0}\right|^{p-2} x_{0}, x_{0}-\bar{x}\right\rangle d s \leq 0 .
\end{aligned}
$$


From the definition of a sup solution of (1.1), we have

$$
\begin{aligned}
& -\int_{A}|D \bar{x}|^{p-2}\left\langle D \bar{x}, D\left(x_{0}+\varepsilon v-\bar{x}\right)\right\rangle d z-\alpha \int_{A}|\bar{x}|^{p-2}\left\langle\bar{x}, x_{0}+\varepsilon v-\bar{x}\right\rangle d z+\int_{A} \bar{u}\left(x_{0}+\varepsilon v-\bar{x}\right) d z \\
& -\int_{\partial A} b(z)|\bar{x}|^{p-2}\left\langle\bar{x}, x_{0}+\varepsilon v-\bar{x}\right\rangle d s \leq 0 .
\end{aligned}
$$

From $A(j)$ (vi), we have $\int_{C} u\left(x_{0}+\varepsilon v\right) d z \leq 0$. Furthermore,

$$
\int_{C}(\bar{u}-u)\left(\bar{x}-x_{0}-\varepsilon v\right) d z \leq \varepsilon c \int_{\left\{x_{0}+\varepsilon v \geq \bar{x}>x_{0}\right\}} v d z .
$$

Also,

$$
m\left\{x_{0}+\varepsilon v \geq \bar{x}>x_{0}\right\} \rightarrow 0, \quad \text { as } \varepsilon \rightarrow 0,
$$

and

$$
\begin{aligned}
& D x_{0}(z)=0 \quad \text { a.e. on }\left\{x_{0}=0\right\}, \\
& D x_{0}(z)=D \bar{x}(z) \quad \text { a.e. on }\left\{x_{0}=\bar{x}\right\} .
\end{aligned}
$$

So, we have

$$
\begin{aligned}
0 \leq & \varepsilon \int_{Z}\left|D x_{0}\right|^{p-2}\left\langle D x_{0}, D v\right\rangle d z+\varepsilon \alpha \int_{Z}\left|x_{0}\right|^{p-2}\left\langle x_{0}, v\right\rangle d z \\
& +\varepsilon \int_{\partial Z} b(z)\left|x_{0}\right|^{p-2}\left\langle x_{0}, v\right\rangle d s-\varepsilon \int_{Z} u v d z \\
& -\varepsilon \int_{C}\left|D x_{0}\right|^{p-2}\left\langle D x_{0}, D v\right\rangle d z+\varepsilon c \int_{\left\{x_{0}+\varepsilon v \geq \bar{x}>x_{0}\right\}} v d z \\
& +\varepsilon \int_{C}\left\langle|D \bar{x}|^{p-2} D \bar{x}-\left|D x_{0}\right|^{p-2} D x_{0}, D v\right\rangle d z .
\end{aligned}
$$

As $\varepsilon \rightarrow 0$, for all $v \in W_{n}^{1, p}(Z)$, we obtain

$$
0 \leq\left\langle I\left(x_{0}\right), v\right\rangle-\int_{Z} u v d z=\left\langle I\left(x_{0}\right)-u, v\right\rangle .
$$

That is,

$$
I\left(x_{0}\right)=u .
$$

Then $x_{0} \in W_{n}^{1, p}(Z)$ is a solution of (1.1).

Step 3: In a similar way, we introduce another truncation function $v_{-}: \mathbb{R} \rightarrow \mathbb{R}_{-}$by

$$
v_{-}(x)= \begin{cases}x, & x<0 \\ 0, & x \geq 0\end{cases}
$$


then define the locally Lipschitz functional $\varphi_{-}: W_{n}^{1, p}(Z) \rightarrow \mathbb{R}$ by

$$
\varphi_{-}(x)=\frac{1}{p}\|D x\|_{p}^{p}+\frac{\alpha}{p}\|x\|_{p}^{p}+\frac{1}{p} \int_{\partial Z} b(z)|x|^{p} d s-\int_{Z} j_{-}(z, x(z)) d z, \quad \forall x \in W_{n}^{1, p}(Z),
$$

where $j_{-}(z, x)=j\left(z, \nu_{-}(x)\right)$ for all $z \in \mathbb{R}, x \in \mathbb{R}$ which is locally Lipschitz. Then we have another nontrivial solution $x_{*} \in W_{n}^{1, p}(Z)$ which is a local minimum of $\varphi_{-}$and of $\varphi$ too.

\section{Existence of the third nontrivial solution}

In this section, we prove the existence of the third solution. Then we give the new assumptions which differ slightly from $A(j)(\mathrm{v})$ :

$A^{\prime}(j) \quad$ (i) $z \mapsto j(z, x)$ is measurable for all $x \in \mathbb{R}$;

(ii) $x \mapsto j(z, x)$ is locally Lipschitz for a.e. $z \in Z$;

(iii) $|u| \leq \gamma(z)+C|x|^{p-1}$ for a.e. $z \in Z$, all $x \in \mathbb{R}, u \in \partial j(z, x)$, with $\gamma \in L^{\infty}(Z)_{+}$and $C>0$;

(iv) $\lim \sup _{|x| \rightarrow \infty} \frac{u}{|x|^{p-2} x} \leq \omega(z)$ for a.e. $z \in Z$, all $u \in \partial j(z, x)$, with $\omega \in L^{\infty}(Z)_{+}$ satisfying $\omega(z) \leq \alpha$ a.e. in $Z$ and $\omega(z)<\alpha$ in some set of positive measure;

(v) $\eta(z) \leq \liminf _{x \rightarrow 0} \frac{u}{\left.|x|\right|^{p-2} x}$ for a.e. $z \in Z$, all $u \in \partial j(z, x)$, with $\eta \in L^{\infty}(Z)_{+}$ satisfying $\lambda_{1}+\alpha \leq \eta(z)$ a.e. in $Z$ and $\lambda_{1}+\alpha<\eta(z)$ in some set of positive measure, $\lambda_{1}$ is the first eigenvalue of $-\Delta_{p}$ with the Robin boundary condition;

(vi) $u x \geq 0$ for a.e. $z \in Z$, all $x \in \mathbb{R}, u \in \partial j(z, x)$.

Theorem 4.1 Let assumptions $A^{\prime}(j)(\mathrm{i})$-(vi) hold. Then we can find three nontrivial solutions $x_{0} \in \operatorname{int}\left(C_{+}\right), x_{*} \in-\operatorname{int}\left(C_{+}\right)$, and $y_{0} \in C_{n}^{1}(\bar{Z})$ of $(1.1)$.

Proof From Theorem 3.1, we have two constant sign solutions $x_{0} \in \operatorname{int}\left(C_{+}\right)$and $x_{*} \in$ $-\operatorname{int}\left(C_{+}\right)$which are the local minimizers of $\varphi_{+}$and of $\varphi_{-}$, also of $\varphi$. We may assume that $x_{0}$ is the only nontrivial critical point of $\varphi_{+}$and $x_{*}$ is the only nontrivial critical point of $\varphi_{-}$. In fact, if there exists another nontrivial critical point $x_{1}$ of $\varphi_{+}, x_{1} \neq x_{0}$. Then, by a similar discussion, $x_{1} \in \operatorname{int}\left(C_{+}\right)$and it solves (1.1). Thus we have a third nontrivial solution, a.e. $y_{0}=x_{1}$.

Moreover, as for $\varphi$, we see that $\varphi$ is coercive and so we can easily prove the Palais-Smale condition. In fact, as in the proof of Theorem 3.1, for a.e. $z \in Z$, all $x \in \mathbb{R}$, we have

$$
j(z, x)<\frac{1}{p}(\omega(z)+\varepsilon)|x|^{p}+\delta_{\varepsilon}(z)|x|
$$

where $\omega$ satisfies (iii), $\delta_{\varepsilon} \in L^{\infty}(Z)_{+}, \delta_{\varepsilon} \neq 0$.

Then, using Lemma 3.5, we have

$$
\begin{aligned}
\varphi(x) & \geq \frac{1}{p}\|D x\|_{p}^{p}+\frac{\alpha}{p}\|x\|_{p}^{p}+\frac{1}{p} \int_{\partial Z} b(z)|x|^{p} d s-\frac{1}{p} \int_{Z} \omega(z)|x(z)|^{p} d z-\frac{\varepsilon}{p}\|x\|_{p}^{p}-C\|x\|_{p} \\
& \geq \frac{1}{p}\left(\varepsilon_{0}-\varepsilon\right)\|x\|_{p}^{p}-C\|x\|_{p} .
\end{aligned}
$$

It follows that $\varphi$ is coercive. 
We set $S=\partial B_{\delta}\left(x_{0}\right)=\left\{x \in W_{n}^{1, p}(Z):\left\|x-x_{0}\right\|_{W_{n}^{1, p}(Z)}=\delta\right\}, Q=\left[x_{*}, x_{0}\right]$, with relative boundary $\partial Q=\left\{x_{*}, x_{0}\right\}$. If we choose $0<\delta<\left\|x_{*}-x_{0}\right\|_{W_{n}^{1, p}(Z)}$. Then $S$ and $\partial Q$ link. In fact, $S \cap \partial Q=\emptyset$, and for any map $h \in C^{0}\left(Q, W_{n}^{1, p}(Z)\right)$ such that $\left.h\right|_{\partial Q}=\mathrm{Id}$, we can choose some $t \in(0,1)$ satisfying

$$
\left\|h\left(t x_{*}+(1-t) x_{0}\right)-x_{0}\right\|_{W_{n}^{1, p}(Z)}=\delta
$$

so $h(Q) \cap S \neq \emptyset, S$ and $\partial Q$ link.

When we choose $\delta$, we can also assume $\delta$ satisfy $\inf _{x \in S} \varphi>\varphi\left(x_{0}\right)$ and $\inf _{x \in S} \varphi>\varphi\left(x_{*}\right)\left(x_{0}\right.$, $x_{*}$ are local minimizers of $\left.\varphi\right)$, we may assume that $\varphi\left(x_{*}\right)<\varphi\left(x_{0}\right)$. Therefore, we can apply Proposition 2.3; let $\Gamma=\left\{h \in C^{0}\left(Q, W_{n}^{1, p}(Z)\right):\left.h\right|_{\partial Q}=\mathrm{Id}\right\}$, produce $y_{0} \in W_{n}^{1, p}(Z)$, a critical point of $\varphi$, such that

$$
\begin{aligned}
& 0 \in \partial \varphi\left(y_{0}\right), \\
& \varphi\left(x_{*}\right)<\varphi\left(x_{0}\right)<\inf _{x \in S} \varphi \leq \varphi\left(y_{0}\right)=\inf _{h \in \Gamma} \sup _{x \in Q} \varphi(h(x)) .
\end{aligned}
$$

From the above inequality, we have $y_{0} \neq x_{0}, y_{0} \neq x_{*}$.

From $0 \in \partial \varphi\left(y_{0}\right)$, we know that

$$
\begin{cases}-\Delta_{p} y_{0}(z)+\alpha\left|y_{0}(z)\right|^{p-2} y_{0}(z) \in \partial j\left(z, y_{0}(z)\right), & z \in Z, \\ \left|\nabla y_{0}(z)\right|^{p-2} \frac{\partial y_{0}(z)}{\partial n}+b(z)\left|y_{0}(z)\right|^{p-2} y_{0}(z)=0, & z \in \partial Z,\end{cases}
$$

and from the regularity theory (see [24]), we have $y_{0} \in C_{n}^{1}(\bar{Z})$, hence (4.1) holds in all $z \in Z$, we get $y_{0} \in C_{n}^{1}(\bar{Z})$.

Finally, we prove that $y_{0} \neq 0$. It is equivalent to proving that there is a path $h \in \Gamma$ such that for all $x \in Q$,

$$
\varphi(h(x))<0=\varphi(0) .
$$

From Proposition 2.1, recall that $S=W_{n}^{1, p}(Z) \cap \partial B_{1}, \partial B_{1}=\left\{x \in L^{p}(Z):\|x\|_{p}=1\right\}$ endowed with the $W_{n}^{1, p}(Z)$-topology. Furthermore, set $S_{c}=S \cap C_{n}^{1}(\bar{Z})$ equipped with the $C_{n}^{1}(\bar{Z})$ topology. Then we can find $h_{0} \in S_{c}$ by virtue of the density of $S_{c}$ in $S$ in the $W_{n}^{1, p}(Z)$ topology, so $C\left(Q, S_{c}\right)$ is dense in $C(Q, S)$, and

$$
\max \left\{\|D x\|_{p}^{p}+\int_{\partial Z} b(z)|x|^{p} d s, x \in h_{0}(Q)\right\} \leq \lambda_{1}+\delta .
$$

From assumption $A^{\prime}(j)(\mathrm{v})$, we can find $\delta_{0}>0$, such that for a.e. $z \in Z$, all $0<|x|<\delta_{0}, u \in$ $\partial j(z, x)$, we get

$$
\eta(z) \leq \frac{u}{|x|^{p-2} x} .
$$

So for a.e. $z \in Z$, all $0<|x|<\delta_{0}$,

$$
\frac{\eta}{p}|x|^{p}<j(z, x)
$$


Since $h_{0}(Q) \in S_{c}$, for the $\delta_{0}$, we can find $\varepsilon>0$, such that for a.e. $z \in \bar{Z}, x \in h_{0}(Q)$, we have

$$
\varepsilon|x(z)| \leq \delta_{0}
$$

Then let $\delta>0$ be such that $\lambda_{1}+\alpha+\delta<\eta$, from (4.2), (4.3), (4.4), and $\|x\|_{p}=1$, we have

$$
\begin{aligned}
\varphi(\varepsilon x) & =\frac{\varepsilon^{p}}{p}\|D x\|_{p}^{p}+\frac{\alpha \varepsilon^{p}}{p}\|x\|_{p}^{p}+\frac{\varepsilon^{p}}{p} \int_{\partial Z} b(z)|x|^{p} d s-\int_{Z} j(z, \varepsilon x(z)) d z \\
& \leq \frac{\varepsilon^{p}}{p}\|D x\|_{p}^{p}+\frac{\alpha \varepsilon^{p}}{p}\|x\|_{p}^{p}+\frac{\varepsilon^{p}}{p} \int_{\partial Z} b(z)|x|^{p} d s-\frac{\eta \varepsilon^{p}}{p}\|x\|_{p}^{p} \\
& =\frac{\varepsilon^{p}}{p}\left(\|D x\|_{p}^{p}+\int_{\partial Z} b(z)|x|^{p} d s\right)+\frac{\alpha-\eta}{p} \varepsilon^{p} \\
& \leq \frac{\lambda_{1}+\delta+\alpha-\eta}{p} \varepsilon^{p}<0 .
\end{aligned}
$$

We consider the continuous path $h_{\varepsilon}=\varepsilon h_{0}$, then for all $x \in Q$,

$$
\varphi\left(h_{\varepsilon}(x)\right)<0, \quad \forall x \in Q
$$

Next recall that $\varphi$ is coercive and satisfies the Palais-Smale condition. From the discussion, we set $a=\varphi\left(x_{0}\right)=\inf \varphi<0, b=0, \varphi$ has no critical points in $\varphi^{-1}(a, b), K_{a}=\left\{x_{0}\right\}$. Then with the help of Proposition 2.5, there exists a deformation $\Phi:[0,1] \times \varphi^{b} \rightarrow \varphi^{b}$ such that

$$
\begin{aligned}
& \left.\Phi(t, \cdot)\right|_{K_{a}}=\mathrm{Id}, \quad \forall t \in[0,1], \\
& \Phi\left(1, \varphi^{b}\right) \subseteq \varphi^{a} \cup K_{a}, \\
& \varphi(\Phi(t, x)) \leq \varphi(x), \quad \forall(t, x) \in[0,1] \times \varphi^{b} .
\end{aligned}
$$

In fact, the continuous path $\Gamma$ can be seen as $\Gamma=\left\{h \in C^{0}\left([0,1], W_{n}^{1, p}(Z)\right): h(0)=\right.$ $\left.x_{*}, h(1)=x_{0}\right\}$. Then we define $h_{1}:[0,1] \rightarrow \varphi^{b}$ by

$$
h_{1}(t)=\Phi\left(t, \varepsilon u_{1}\right), \quad \forall t \in[0,1] .
$$

Then it is a continuous path, so from (4.5), we have

$$
\begin{aligned}
& h_{1}(0)=\Phi\left(0, \varepsilon u_{1}\right)=\varepsilon u_{1}, \\
& h_{1}(1)=\Phi\left(1, \varepsilon u_{1}\right)=x_{0} \quad\left(\varphi^{a}=\emptyset, K_{a}=\left\{x_{0}\right\}\right), \\
& \varphi\left(h_{1}(t)\right)=\varphi\left(\Phi\left(t, \varepsilon u_{1}\right)\right) \leq \varphi\left(\varepsilon u_{1}\right)<0, \quad \forall t \in[0,1]\left(\left.\varphi\right|_{h_{\varepsilon}}<0\right) .
\end{aligned}
$$

Thus, we construct a continuous path $h_{1}$ joining $\varepsilon u_{1}$ and $x_{0}$ such that

$$
\left.\varphi\right|_{h_{1}}<0
$$

Similarly, we construct a continuous path $h_{2}$ joining $-\varepsilon u_{1}$ and $x_{*}$ such that

$$
\left.\varphi\right|_{h_{2}}<0 .
$$


Then we join $h_{2}, h_{\varepsilon}, h_{1}$, and we construct a continuous path $h \in \Gamma$ such that

$$
\left.\varphi\right|_{h}<0 \text {. }
$$

It follows that $\varphi\left(y_{0}\right)<0=\varphi(0)$ and so $y_{0} \neq 0$.

Therefore, we find the third nontrivial solution of (1.1).

\section{Open related questions}

\section{Consider the problem}

$$
\left\{\begin{array}{l}
-\Delta_{p} x+\alpha(z)|x|^{p-2} x \in \partial j(z, x), \quad z \in Z \\
|\nabla x|^{p-2} \frac{\partial x}{\partial n}+b(z)|x|^{p-2} x=0, \quad z \in \partial Z
\end{array}\right.
$$

where $Z \subset \mathbb{R}^{N}$ is a bounded domain with $C^{2}$-boundary $\partial Z, \Delta_{p} x=\operatorname{div}\left(|\nabla x|^{p-2} \nabla x\right)(1<p<$ $\infty)$ is the $p$-Laplacian operator, $\alpha(z), b(z) \in L^{\infty}(\partial Z), b(z) \geq 0$, and $b(z) \neq 0$ on $\partial Z$. $j(z, x)$ is a measurable potential function on $Z \times \mathbb{R}$, which is locally Lipschitz in the $x \in \mathbb{R}, \partial j(z, x)$ stands for the generalized subdifferential of $x \mapsto j(z, x)$. Also $\frac{\partial x}{\partial n}$ denotes the outer normal derivative of $x$ with respect to $\partial Z$.

Whether problem (5.1) has more solutions and whether it has oscillating solutions, we will discuss in the future.

\section{Competing interests}

The author declares that she has no competing interests.

\section{Author's contributions}

The author wrote, read, and approved the final manuscript.

\section{Acknowledgements}

The author was supported by Tianyuan Foundation for Mathematics under Grant No. 11326098 of National Natural Science Foundation and Doctor Scientific Research Startup Foundation of Harbin Normal University No. XKB 201311.

Received: 5 January 2014 Accepted: 1 December 2014 Published online: 20 December 2014

\section{References}

1. Ambrosetti, A, Garcia Azorero, J, Peral Alnoso, I: Multiplicity results for some nonlinear elliptic equations. J. Funct. Anal. $137,219-242(1996)$

2. Garcia Azorero, JP, Peral Alnoso, I, Manfredi, JJ: Sobolev versus Höder local minimizers and global multiplicity for some quasilinear elliptic equations. Commun. Contemp. Math. 2, 385-404 (2000)

3. Zhang, J, Li, SJ, Xue, XP: Multiple solutions for a class of semi-linear elliptic Robin problem. J. Math. Anal. Appl. 388 , 435-442 (2012)

4. Zhang, J, Li, C, Xue, XP: Nontrivial solutions for a class of quasilinear problems with jumping nonlinearities via a co-homological local splitting. Nonlinear Anal. 75, 4896-4903 (2012)

5. Zhang, J, Xue, XP: Multiple solutions of $p$-Laplacian with Neumann and Robin boundary conditions for both resonance and oscillation problem. Bound. Value Probl. 2011, Article ID 214289 (2011)

6. Anello, G: Existence of infinitely many weak solutions for a Neumann problem. Nonlinear Anal. 57, 199-209 (2004)

7. Ricceri, B: Infinitely many solutions for the Neumann problem for elliptic equations involving the $p$-Laplacian. Bull. Lond. Math. Soc. 33, 331-340 (2001)

8. Barletta, G, Papageorgiou, NS: A multiplicity theorem for the Neumann $p$-Laplacian with an asymmetric nonsmooth potential. J. Glob. Optim. 39, 365-392 (2007)

9. Dancer, EN, Du, Y: On sign-changing solutions of certain semilinear elliptic problems. Appl. Anal. 56, 193-206 (1995)

10. Corvellec, JN: On the second deformation lemma. Topol. Methods Nonlinear Anal. 17, 55-66 (2001)

11. Carl, S, Perera, K: Sign-changing and multiple solutions for p-Laplacian. Abstr. Appl. Anal. 7, 613-625 (2002)

12. Iannizzotto, A, Papageorgiou, NS: Existence of three nontrivial solutions for nonlinear Neumann hemivariational inequalities. Nonlinear Anal. 70, 3285-3297 (2009)

13. Agarwal, RP, et al.: Constant sign and nodal solutions for problems with the $p$-Laplacian and a non-smooth potential using variational techniques. Bound. Value Probl. 2009, Article ID 820237 (2009)

14. Gasinski, L, Papageorgiou, NS: Nonsmooth Critical Point Theory and Nonlinear Boundary Value Problems. Series in Mathematical Analysis and Applications, vol. 8. Chapman \& Hall/CRC, Boca Raton (2005)

15. Lindqvist, P: On the equation $\operatorname{div}\left(|\nabla u|^{p-2} \nabla u\right)+\lambda|u|^{p-2} u=0$. Proc. Am. Math. Soc. 109(1), 157-164 (1990) 
16. Di Benedetto, $\mathrm{E}: \mathrm{C}^{1+\alpha}$ Local regularity of weak solutions of degenerate elliptic equations. Nonlinear Anal. 7, 827-850 (1983)

17. Di Benedetto, E: Partial Differential Equations. Birkhäuser, Boston (1995)

18. Vazquez, JL: A strong maximum principle for some quasilinear elliptic equations. Appl. Math. Optim. 12, 191-202 (1984)

19. Aizicovici, S, Papageorgiou, NS, Staicu, V: The spectrum and an index formula for the Neumann $p$-Laplacian and multiple solutions for problems with crossing nonlinearity. Discrete Contin. Dyn. Syst. 25(2), $431-456$ (2009)

20. Struwe, M: Variational Methods. Springer, Berlin (1990)

21. Hatcher, A: Algebraic Topology. Cambridge University Press, Cambridge (2001)

22. Clarke, FH: Optimization and Nonsmooth Anal. SIAM, Philadelphia (1990)

23. Renardy, M, Rogers, RC: An Introduction to Partial Differential Equations, 2nd edn. Texts in Applied Mathematics, vol. 13, pp. 367. Springer, New York (2010)

24. Gasinski, L, Papageorgiou, NS: Nonlinear Analysis. Series in Mathematical Analysis and Applications, vol. 9. Chapman \& Hall/CRC, Boca Raton (2006)

25. Chang, KC: Variational methods for nondifferentiable functionals and their applications to partial differential equations. J. Math. Anal. Appl. 80, 102-129 (1981)

doi:10.1186/s13661-014-0257-5

Cite this article as: Zhang: Existence of solutions for nonlinear Robin problems with the $p$-Laplacian and hemivariational inequality. Boundary Value Problems 2014 2014:257.

\section{Submit your manuscript to a SpringerOpen ${ }^{\circ}$ journal and benefit from:}

- Convenient online submission

- Rigorous peer review

- Immediate publication on acceptance

- Open access: articles freely available online

- High visibility within the field

- Retaining the copyright to your article 